\title{
Wide complex tachycardia with atrioventricular dissociation and QRS morphology identical to that of sinus rhythm: a manifestation of bundle branch reentry
}

Giuseppe Oreto, Joep L R M Smeets, Luz-Maria Rodriguez, Carl Timmermans, Hein J J Wellens

\begin{abstract}
Objective-To determine the features that distinguish bundle branch reentry (BBR) ventricular tachycardia from a supraventricular tachycardia with aberration on the 12 lead electrocardiogram (ECG).

Patients-Three patients in whom premature beats ( 2 cases) or sustained tachycardia ( 2 cases) showed a QRS configuration identical to that observed during sinus rhythm.

Interventions-Programmed electrical stimulation.

Results-These arrhythmias were ventricular in origin and caused by a BBR mechanism, as suggested by the following data obtained during electrophysiological study:
\end{abstract}

(a) an $\mathrm{H}-\mathrm{V}$ interval shorter during tachycardia than during sinus rhythm; (b) A-V dissociation; (c) activation of the right bundle branch before activation of the bundle of His. The ECG of all 3 patients showed right bundle branch block with very prolonged QRS duration $(0.16$ to $0.20 \mathrm{~s})$. Characteristically, all 3 had prolonged $\mathrm{H}-\mathrm{V}$ interval during sinus rhythm. All patients had had a previous myocardial infarction and had a dilated left ventricle.

Conclusion-The presence of (a) wide complex extrasystoles or tachycardia with a QRS morphology identical to that of sinus rhythm; (b) A-V dissociation; and (c) a very prolonged $Q R S$ duration $(0.16 \mathrm{~s}$ or more) is suggestive of ventricular tachycardia caused by bundle branch reentry.

Cardiology, Academic

Hospital, Maastricht,

The Netherlands

G Oreto

J L R M Smeets

L-M Rodriguez

C Timmermans

H J J Wellens

Correspondence to:

Dr G Oreto, Department of

Cardiology, Academic

Cardiology, Academic

P.O. Box 5800, 6202 AZ

Maastricht,

The Netherlands.

Accepted for publication 18 July 1996

\section{(Heart 1996;76:541-547)}

Keywords: bundle branch reentry; ventricular tachycardia; wide QRS complex tachycardia

A wide QRS tachycardia can be caused by (a) a supraventricular tachycardia with functional aberrant conduction or pre-existing bundle branch block, (b) a supraventricular tachycardia with atrioventricular (A-V) conduction over an accessory pathway, or (c) impulse formation originating in the ventricle. Comparison of the electrocardiogram (ECG) during sinus rhythm and during tachycardia usually allows ventricular tachycardia (VT) to be distinguished from supraventricular tachycardia (SVT). An identical QRS morphology during tachycardia and sinus rhythm strongly suggests a supraventricular origin of the arrhythmia. ${ }^{12}$ However, occasionally a VT may show a QRS configuration identical to that during sinus rhythm because the arrhythmia is caused by bundle branch reentry (BBR).

We report three cases in which QRS complexes with the same morphology as during sinus rhythm were present during a ventricular arrhythmia, and we discuss the criteria that can lead to the correct diagnosis.

\section{Case reports}

CASE 1

Figure 1 shows the electrocardiogram during sinus rhythm (A) and wide complex tachycardia (B) recorded from a 74 year old man who had had an anterior myocardial infarction with subsequent ventricular aneurysm formation several years before admission. The patient was studied to evaluate his sub A-V nodal conduction and episodes of wide QRS tachycardia. He was not taking any antiarrhythmic drugs when he was studied.

The tracing in sinus rhythm showed left atrial enlargement, a prolonged $\mathrm{P}-\mathrm{R}$ interval $(0.24 \mathrm{~s})$, wide $\mathrm{QRS}$ complexes $(0.16 \mathrm{~s})$, and right axis deviation. The ventricular complexes did not have the morphology that is typical of simple bundle branch block, although the limb leads and V6 suggested a right bundle branch block (RBBB) and left posterior hemiblock. There were, in addition, signs of myocardial infarction suggested by a deep and broad q wave in lead V1 and small q waves in leads V2 and V3.

The right panel of fig 1 shows a wide complex tachycardia with a rate of 160 per minute and a QRS configuration identical to that seen during sinus rhythm. This could suggest, at first glance, a supraventricular origin of the tachycardia. However, a pattern of $\mathrm{A}-\mathrm{V}$ disso- 


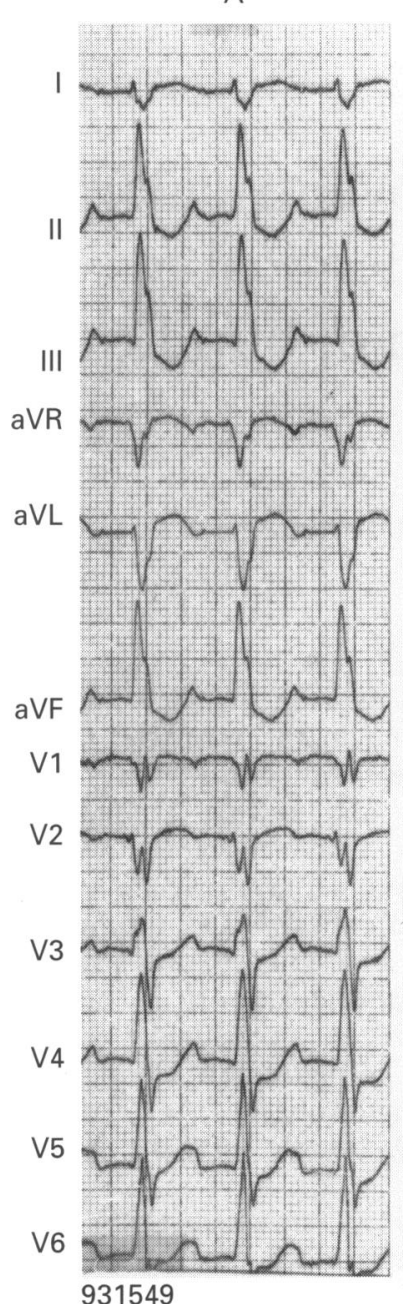

B
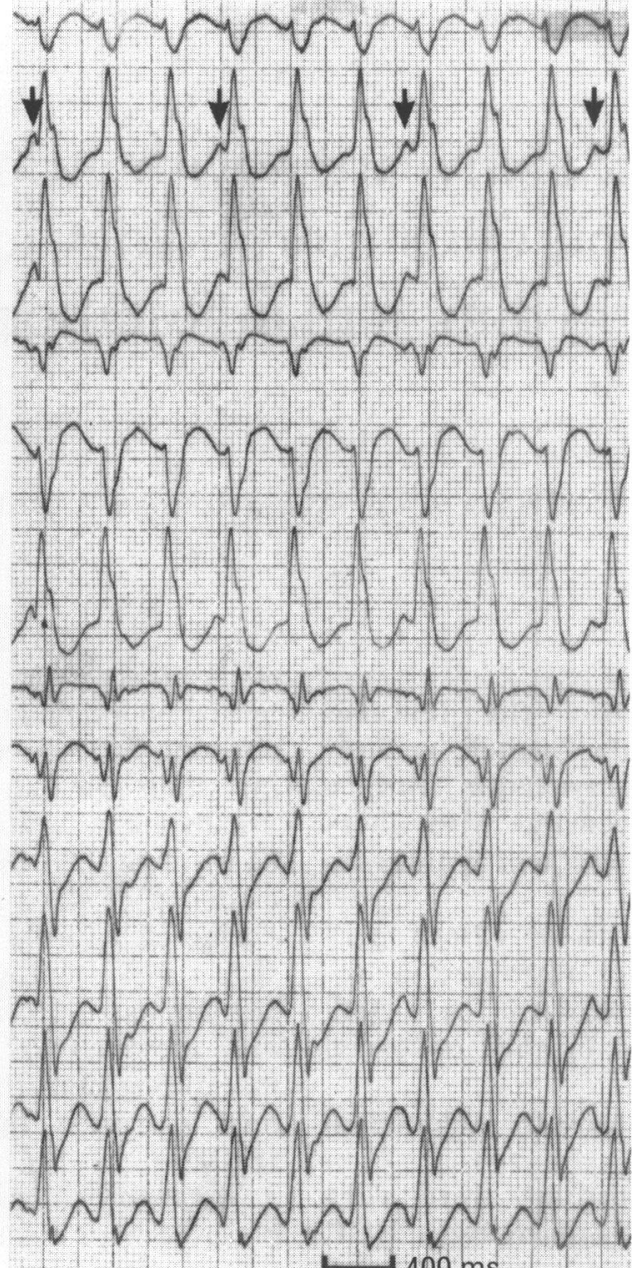

Figure 1 Electrocardiograms recorded during sinus rhythm $(A)$ and tachycardia $(B)$ in case 1. Arrows indicate independent sinus $P$ waves. ciation was present, which was evident in lead II, where upright $P$ waves with a rate the same as sinus rhythm were identified (arrows).

Figure 2 (panel A) shows that during sinus rhythm the $\mathrm{A}-\mathrm{H}$ and $\mathrm{H}-\mathrm{V}$ intervals were prolonged, measuring 135 and $70 \mathrm{~ms}$, respectively. The tracing recorded during tachycardia (panel B) shows that every ventricular complex was preceded by an $\mathrm{H}$ deflection with an interval of $50 \mathrm{~ms}$, which is $20 \mathrm{~ms}$ shorter than the $\mathrm{H}-\mathrm{V}$ interval during sinus rhythm. In addition, A-V dissociation was present. The patient was successfully treated with RBB ablation followed by permanent peacemaker implantation.

\section{CASE 2}

Figure 3 shows the 12 lead ECG during sinus tachycardia at a rate of 120 per minute (A) and during paroxysmal tachycardia at a rate of 175 per minute (B) from a 63 year old man with a history of an old myocardial infarction, congestive heart failure, and recurrent episodes of tachycardia. At admission the patient was not taking any antiarrhythmic drugs. He was initially treated unsuccessfully with intravenous procainamide and then underwent an electrophysiological study. During sinus tachycardia the P-R interval was prolonged $(0.21 \mathrm{~s})$, with $P$ waves that were partially superimposed upon the preceding $\mathrm{T}$ waves, but clearly visible in leads I and V3. The QRS was wide $(0 \cdot 19 \mathrm{~s})$, and showed a morphology suggestive of RBBB plus left anterior hemiblock and a possible old anteroseptal myocardial infarction. The third complex in panel A was an extrasystole that was identical in morphology to sinus beats, and was dissoci-
Figure 2 A simultaneous recording of the 12 lead ECG and the His bundle recording during sinus rhythm $(A)$ and tachycardia $(B)$ in case 1.
A

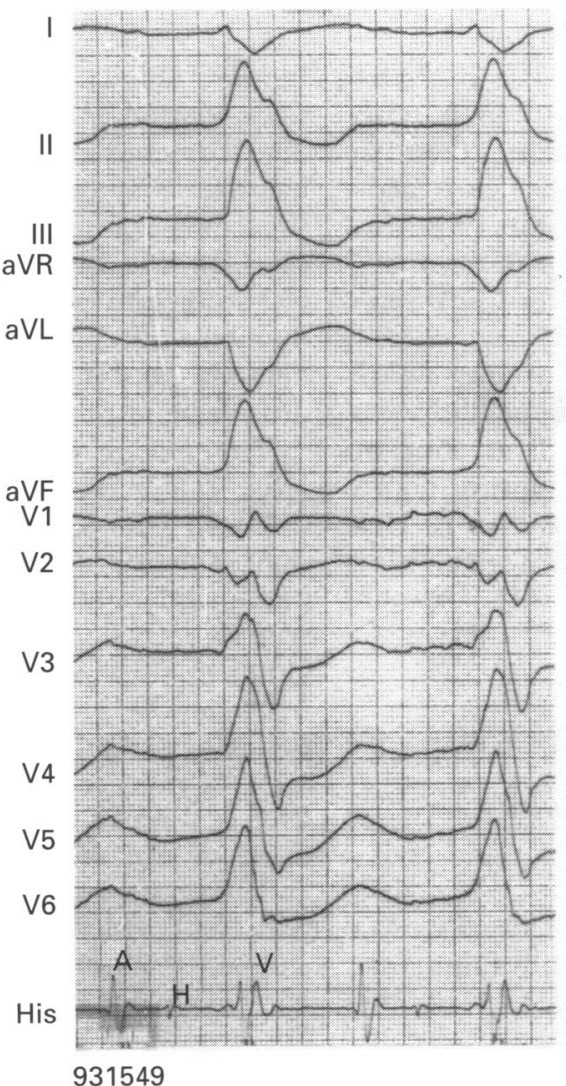

B 93868 
Figure 3

Electrocardiograms recorded during sinus tachycardia

(A) and paroxysmal tachycardia (B) in case 2. Arrows in section $B$ point to sinus $P$ waves which are dissociated from the $Q R S$ complexes.
Figure 4 Seven simultaneously recorded surface leads and intracardiac recordings during sinus rhythm $(A)$ and tachycardia $(B)$ in case 2. $H B E d$ and $H B E p$, distal and proximal $\mathrm{His}$ bundle electrograms; LRA, low right atrium; $L V$, left ventricle; $H B E$, $H$ is bundle electrogram; $L B$, left bundle branch potential; $H$, His bundle potential; $R B$, right bundle branch potential.



$4.400 \mathrm{~ms} 4 \mathrm{t}$ ated from the preceding sinus $\mathrm{P}$ wave. The sinus rhythm was not disturbed by the extrasystole, and the postextrasystolic pause permitted a detailed analysis of the $P$ wave, which had a configuration that indicated left atrial enlargement. The paroxysmal tachycardia (panel B) had exactly the same QRS configuration as during sinus rhythm, but showed
A-V dissociation. The arrows indicate the sinus $\mathrm{P}$ waves, which are clearly recognisable in lead I. After the fourth beat, which was premature, tachycardia terminated and a pause occurred. Two conducted sinus beats followed, and tachycardia restarted again.

Figure 4 shows the intracavitary recordings. During sinus rhythm (A), the $\mathrm{H}-\mathrm{V}$ interval was
A

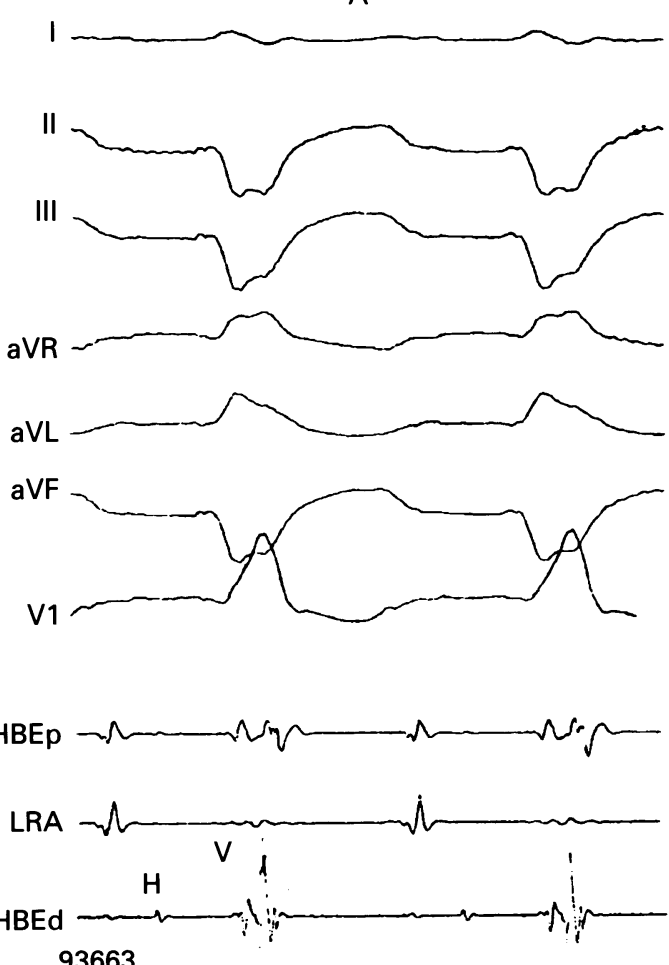

B
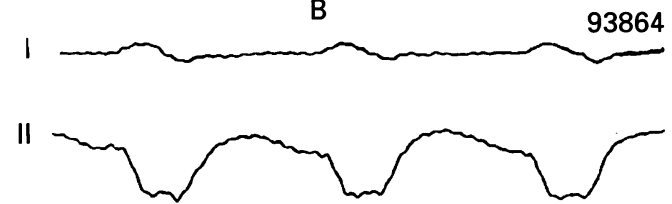

III
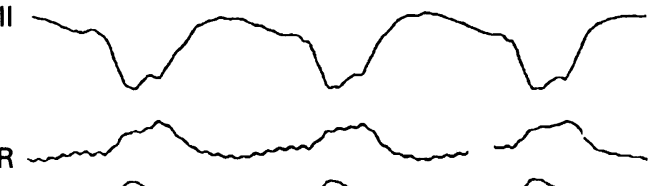

aVL



aVF

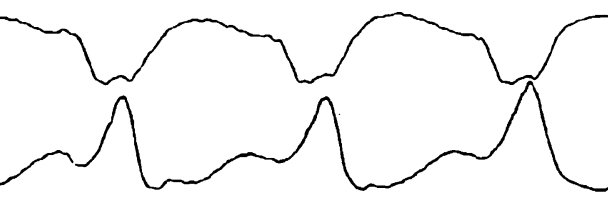

LV

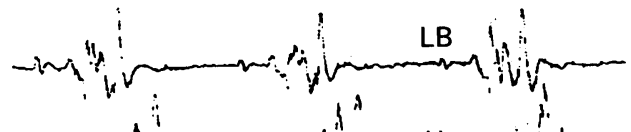
HBE

RV .r.sing 




Figure 5 Twelve lead ECG in case 3 showing extrasystoles in a trigeminal rhythm.

Figure 6 Seven simultaneously recorded surface leads and intracardiac recordings during sinus rhythm and a ventricular premature beat in case 3 . HRA, high right atrium; HBEd and $H B E p$, distal and proximal His bundle electrograms.
$80 \mathrm{~ms}$. During tachycardia (B) every QRS complex was preceded by an $\mathrm{H}$ deflection with an $\mathrm{H}-\mathrm{V}$ interval of $65 \mathrm{~ms}$. In the attempt to clarify the mechanism of tachycardia, catheters were introduced into the right and the left ventricles. The right ventricular catheter was advanced across the tricuspid valve, anteriorly and superiorly to the His catheter, in order to record the RBB potential. The left ventricular catheter was placed along the superior region of the septum in order to record the LBB potential. Recordings from the right and left ventricle showed that the RBB potential preceded the $\mathrm{H}$ deflection whereas the left bundle branch potential followed it.

Analysis of the relation between the $\mathrm{H}-\mathrm{H}$ and $\mathrm{V}-\mathrm{V}$ intervals revealed a precise correlation between the $\mathrm{H}-\mathrm{H}$ and the ensuing $\mathrm{V}-\mathrm{V}$, whereas no relation existed between the $\mathrm{H}-\mathrm{H}$ and the preceding $\mathrm{V}-\mathrm{V}$ interval.

In this patient, tachycardia was nearly incessant; whenever the arrhythmia terminated, it almost invariably resumed after one or a few sinus beats which were conducted to the ventricles. Catheter ablation of the RBB was attempted, but it was only partially successful. Tachycardia recurred six hours later and the patient went into cardiogenic shock and died.

\section{CASE 3}

Figure 5 shows the electrocardiogram recorded from a 71 year old man who had had an old anterior myocardial infarction and was admitted for recurrent sustained VT. The tracing showed sinus rhythm at a rate of 70 beats per minute, left atrial enlargement, prolonged P-R interval ( $0.24 \mathrm{~s}), \mathrm{RBBB}$ with $\mathrm{QRS}$ duration of $0.20 \mathrm{~s}$, and anterior myocardial infarction, as revealed by deep and broad $Q$ waves in leads V1 to V3. Trigeminal extrasystoles also occurred. They were characterised by premature QRS complexes with a configuration that was almost identical to that of sinus beats, with very minor differences represented by deeper S waves in leads III, aVF, V4, V5, and V6, and taller $R$ waves in lead aVL. Extrasystoles were not preceded by a $P$ wave, and were dissociated from the sinus $P$ waves, which occurred in step with the sinus rate, and deformed the $\mathrm{T}$ waves of the premature complexes. 

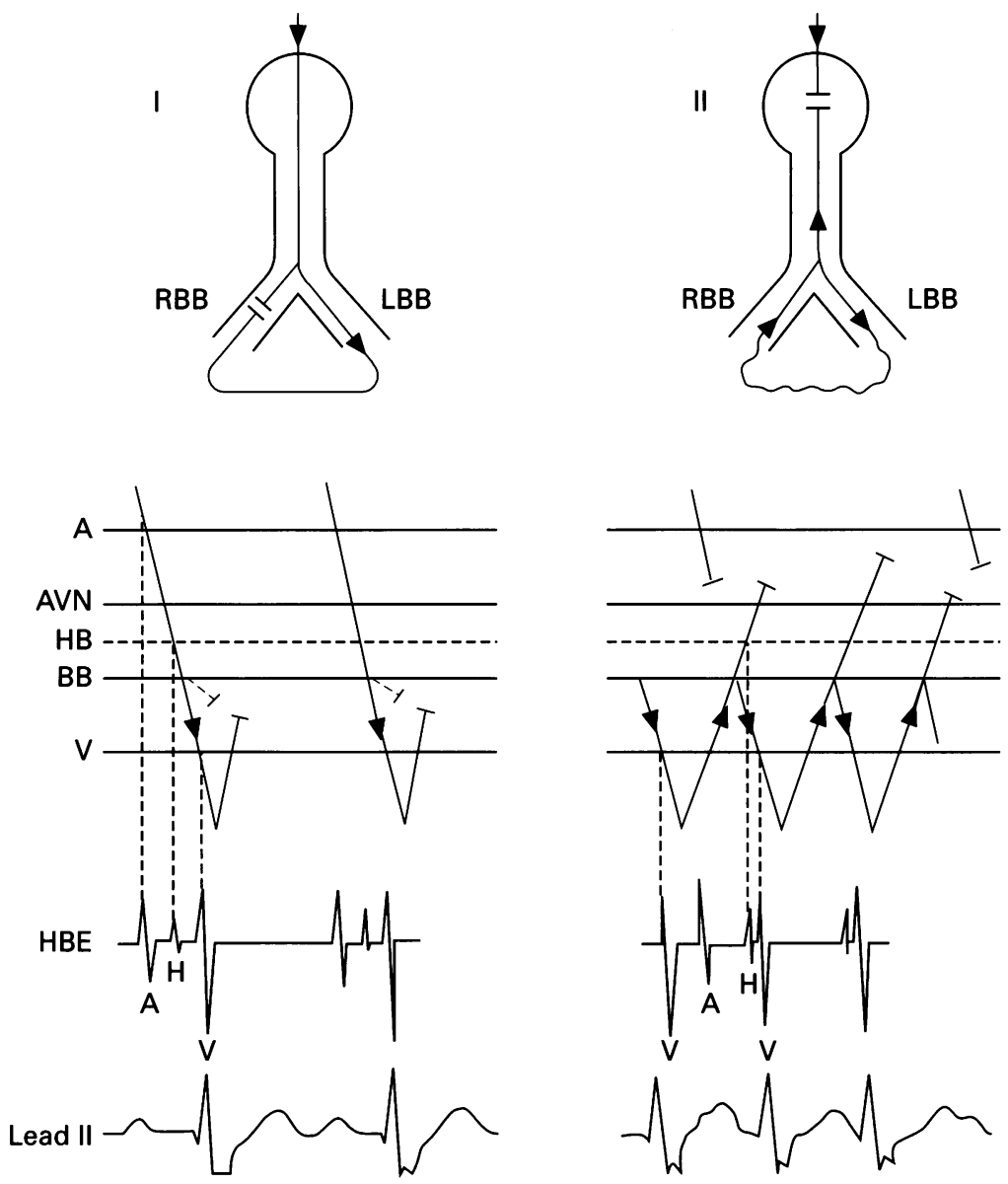

Figure 7 Diagram I shows sinus rhythm with right bundle branch block and diagram II bundle branch reentry tachycardia with anterograde conduction over the left bundle branch. Every scheme contains, from top to bottom: a drawing showing the $A-V$ node and the His bundle with the bundle branches, a ladder diagram, a His bundle electrogram (HBE), and a surface electrocardiogram (lead II). In the ladder diagram the atria $(A)$, the $A-V$ node $(A V N)$, the His bundle $(H B)$, the bundle branches $(B B)$, and the ventricles $(V)$ are represented. The horizontal broken line in the $H B$ layer indicates the site of recording of the His bundle potential. In the BB section conduction through the $L B B$ is represented with a solid line and conduction through the $R B B$ with a broken line. See text.

Figure 6 shows that the $\mathrm{H}-\mathrm{V}$ interval of sinus beats was prolonged $(80 \mathrm{~ms})$. Premature beats, in contrast, had a shorter $\mathrm{H}-\mathrm{V}$ interval than the sinus beats $(50 \mathrm{~ms})$. Programmed ventricular stimulation initiated a sustained VT with a configuration different from that of the sinus beats, but non-sustained VT with morphology identical to sinus rhythm was also induced.

\section{Discussion}

The tachycardias of cases 1 and 2 and the extrasystoles of cases 2 and 3 were ventricular and not supraventricular in origin. They were caused by bundle branch reentry, a phenomenon that accounts for the similar morphology of sinus beats and ectopic ventricular beats (see below). Another possibility is that the arrhythmias originated in the ventricles and were caused by focal reentry in a circuit not involving the bundle branches; in such a case it is necessary to assume that the impulse selectively enters the conduction system before being distributed to the rest of the ventricles. Bundle branch reentry (BBR) was suggested as a possible mechanism for VT more than 20 years ago. ${ }^{3}$ In this type of tachycardia the circuit incorporates both bundle branches and the ventricular myocardium connecting the bundle branches. The circulating impulse goes down to the ventricle through one bundle branch and comes up through the contralateral bundle branch..$^{3-8}$ In very rare cases the circuit can involve the two divisions of the left bundle branch. ${ }^{69}$ The ventricular complexes show a bundle branch block configuration because the ventricle is activated by one of the bundle branches.

The diagnosis of BBR VT requires the following findings:

- VT with the configuration of a bundle branch block. ${ }^{356}$

- An $\mathrm{H}-\mathrm{V}$ interval during tachycardia that is shorter than or equal to the $\mathrm{H}-\mathrm{V}$ interval during sinus rhythm.

The explanation for the shorter $\mathrm{H}-\mathrm{V}$ interval during tachycardia is given in fig 7 . Diagram I shows that during sinus rhythm in a patient with $\mathrm{RBBB}$ the impulse traverses anterogradely through the His bundle and the $\mathrm{LBB}$ to reach the ventricle. The $\mathrm{H}-\mathrm{V}$ interval reflects the time required to go from the site of His bundle deflection recording (the broken horizontal line in the HB section of the ladder diagram) to the ventricular myocardium.

Diagram II shows a BBR VT. The impulse reaches the ventricles through the $\mathrm{LBB}$ and retrogradely re-enters the $R B B$. When the impulse coming from the $\mathrm{RBB}$ arrives at the bifurcation, it turns down to re-invade the LBB, and also continues its retrograde course towards the $\mathrm{A}-\mathrm{V}$ node over the $\mathrm{His}$ bundle. The His bundle is activated retrogradely, and the $\mathrm{H}-\mathrm{V}$ interval is shorter than in diagram I because the re-entering impulse penetrates simultaneously into the LBB and the His bundle. This means that the His bundle and the LBB are activated more or less in parallel, whereas during sinus rhythm (diagram I) they are activated in series. A shorter $\mathrm{H}-\mathrm{V}$ interval in BBR VT than is sinus rhythm was reported by Blanck $e t a l^{8}$ in two out of three patients who manifested this type of arrhythmia in the absence of myocardial or valve dysfunction. Cases of BBR VT with an $\mathrm{H}-\mathrm{V}$ interval equal to or longer than that recorded during sinus rhythm, however, have also been reported. ${ }^{5}$ The different relation between the $\mathrm{H}-\mathrm{V}$ interval during tachycardia and in sinus rhythm could depend on catheter location, and also on the degree of anterograde His-Purkinje conduction delay.

- The activation of the bundle branch that is used retrogradely (the RBB in the example of fig 4) precedes the $\mathrm{H}$ potential, whereas the activation of the bundle branch that is used anterogradely (the LBB in fig 4) follows the $\mathrm{H}$ deflection. ${ }^{5}{ }^{6}$

- Changes in V-V intervals are preceded by comparable changes in $\mathrm{H}-\mathrm{H}$ intervals. ${ }^{5}$

A rare but convincing finding for BBR is termination of a VT by a premature atrial impulse that is not conducted to the ventricles. ${ }^{4}$

Bundle branch reentry requires a critical intraventricular delay, otherwise the impulse conducted retrogradely over a bundle branch 
would reach the contralateral bundle branch when the latter is still refractory. In our three cases an old anteroseptal infarction was present. This resulted not only in unidirectional bundle branch block and delayed anterograde conduction in the other bundle branch, as expressed by a prolonged $\mathrm{H}-\mathrm{V}$ interval, but also in a prolonged transseptal conduction time, which is required to allow retrograde penetration and conduction over the anterogradely blocked bundle branch.

Bundle branch reentry is favoured by a preexisting bundle branch block, provided that such a block is unidirectional. Whenever bundle branch block is present in sinus rhythm, the morphology of BBR VT (or BBR extrasystoles) will be identical to that of sinus beats. This is because ventricular activation starts at the exit of the anterogradely conducting bundle branch. This concept is explained in fig 7, which shows that sinus rhythm (panel I) and BBR VT (panel II) share the same QRS morphology.

In the presence of unidirectional bundle branch block BBR can be initiated by a supraventricular impulse whenever this impulse is sufficiently premature to undergo additional delay within the bundle branches or the interventricular septal tissue to complete the reentry cycle. In case 2 BBR VT was repeatedly initiated by sinus beats (fig 3 ) because bundle branch and intraventricular conduction were impaired to such a degree that even a non-premature impulse was able to complete the full reentry cycle.

A BBR VT, as well as any VT, could theoretically be associated with 1:1 retrograde conduction to the atria. In our patients, however, $\mathrm{A}-\mathrm{V}$ dissociation was present. Only one case with 1:1 V-A conduction during BBR VT has been reported. ${ }^{4}$

The diagnosis of BBR VT is difficult on the surface ECG because:

- The similar QRS morphology during VT and sinus rhythm suggests a form of SVT.

- If VT and sinus rhythm have a different QRS morphology, analysis of the QRS configuration will suggest a SVT, because a bundle branch block pattern will be present during VT, and because one ventricle is normally activated by way of the anterogradely conducting bundle branch.

The most important feature favouring the recognition of the ventricular rather than supraventricular origin of tachycardia is A-V dissociation. Atrioventricular dissociation is not definite proof of VT, because it can also occur in A-V junctional tachycardia without retrograde conduction. A definite diagnosis can only be made by intracardiac recordings and programmed electrical stimulation. In A$\mathrm{V}$ junctional tachycardia the $\mathrm{H}-\mathrm{V}$ interval will not be shorter during tachycardia than during sinus rhythm, and the His deflection will precede those of the bundle branches.

Some of the features found in our three patients are unusual. In contrast to most reported cases of BBR VT, ${ }^{6}$ our patients had an RBBB shaped VT rather than a LBBB shaped VT. Also all three patients had had an anteroseptal myocardial infarction and showed slurred QRS complexes and RBBB during sinus rhythm. In one of our patients, almost incessant tachycardia was present because sinus beats already initiated BBR. These findings suggest that in the patients with previous myocardial infarction and bundle branch block the prerequisites for BBR (slowed conduction in the bundle branches and in the scarred intraventricular septum) are present, and BBR might occur more often than presently realised.

Ventricular tachycardia with the same configuration of QRS complexes as in sinus rhythm has been reported by Olshansky ${ }^{10}$ in three patients. All patients had a pattern of RBBB during both sinus rhythm and VT; they also showed marked left axis deviation and had had an anterior myocardial infarction. A$\mathrm{V}$ dissociation was present in every patient. Although no His deflection was recorded in the two patients who underwent electrophysiological examination, it is very likely that the mechanism responsible for VT in the cases reported by Olshansky was BBR.

\section{RECOGNITION OF BUNDLE BRANCH REENTRY} ON THE SURFACE ECG

Intracardiac recordings are necessary for the definite diagnosis of BBR, but the following electrocardiographic features are suggestive of this mechanism.

- In the case of isolated premature beats, BBR is suggested by a premature complex (a) identical or almost identical to sinus beats; (b) dissociated from the sinus $P$ wave; and (c) with very prolonged QRS duration ( $0.16 \mathrm{~s}$ or more), as an expression of bundle branch block and intraventricular conduction delay.

- In the case of wide QRS complexes tachycardia, BBR should be suspected in the presence of (a) a QRS configuration identical to sinus rhythm; (b) A-V dissociation; and (c) a very wide QRS of $0 \cdot 16$ $s$ or more.

Wide QRS complex tachycardias should not automatically be diagnosed as being supraventricular simply because they have a QRS morphology identical to that during sinus rhythm. Such a diagnosis could lead to the wrong treatment with calcium channel blocking drugs, resulting in dramatic worsening of the clinical situation. ${ }^{11}$ Furthermore, recognition of BBR VT is also important because this arrhythmia can be effectively treated with catheter ablation of the RBB. ${ }^{6812}$

We thank Ms Adri van den Dool for her help in the preparation of the illustrations.

1 Wellens HJJ, Conover MB. The ECG in emergency decision making. Philadelphia: WB Saunders, 1992

2 Dongas J, Lehmann MH, Mahmud R, Denker S, Soni I Akhtar $M$. Value of preexisting bundle branch block in the electrocardiographic differentiation of supraventricula from ventricular origin of wide QRS tachycardia. $A m \mathcal{F}$ Cardiol 1985;55:717.

3 Wellens HJJ, Schuilemburg RM, Durrer D. Electrical stimulation of the heart in patients with ventricular tachycar dia. Circulation 1972;46:216-26. 
4 Wellens HJ, Lie KI, Durrer D. Further observations on ventricular tachycardia as studied by electrical stimulation of the heart. Circulation 1974;49:647-53.

5 Caceres J, Jazayeri M, McKinnie J, Avitall B, Denker ST Tchau $\mathrm{P}$, et al. Sustained bundle branch reentry as a mechanism of clinical tachycardia. Circulation 1989 79:256-70

6 Blanck Z, Dhala A, Deshpande S, Sra J, Jazayeri M, Akhtar $M$, et al. Bundle branch reentrant ventricular tachycardia. Cumulative experience in 48 patients. $f$ Cardiovas Electrophysiol 1993;4:253-62.

7 Akhtar M. The clinical spectrum of ventricular tachycardia. Circulation 1990;82:1561-73.

8 Blanck Z, Jazayeri M, Dhala A, Deshpande S, Sra J, Akhtar $M$. Bundle branch reentry: a mechanism of ventricula tachycardia in the absence of myocardial or valvular dysfunction. $\mathcal{F}$ Am Coll Cardiol 1993;22:1718-22.
9 Crijns HJGM, Smeets JLRM, Rodriguez LM, Meijer A, Welens HJJ. Cure of interfascicular reentrant ventricular tachycardia by ablation of the anterior fascicle of the left bundle branch. $f$ Cardiovasc Electrophysiol 1995;6: 486-92.

Olshansky B. Ventricular tachycardia masquerading as supraventricular tachycardia: a wolf in sheep's clothing. $f$ Electrocardiol 1988;21:377-84

11 Buxton AE, Marchlinski FE, Doherty JU, Flores B, Josephson ME. Hazards of intravenous verapamil for sustained ventricular tachycardia. Am $\mathcal{f}$ Cardiol 1987; 59:1107-10.

12 Tchou P, Jazayeri M, Denker S, Dongas J, Caceres J, Akhtar M. Transcatheter electrical ablation of the right bundle branch: a method of treating macro-reentrant ventricular tachycardia due to a bundle branch reentry. Circulation 1988;78:246-57. 